\title{
ВЫБОР ПАРАМЕТРОВ И АНАЛИЗ ЭФФЕКТИВНОСТИ ПРИМЕНЕНИЯ СИСТЕМ СЛЕЖЕНИЯ ЗА СОЛНЦЕМ
}

\author{
Обухов Сергей Геннадьевич', \\ serob99@mail.ru \\ Плотников Игорь Александрович', \\ igorplt@tpu.ru
Национальный исследовательский Томский политехнический университет, Россия, 634050, г. Томск, пр. Ленина, 30.

Актуальность. Эффективным способом повышения производительности фотоэлектрических станций является применение систем слежения за положением Солнца. Ярко-выраженная зависимость величины солнечного излучения, поступающего на приемную поверхность солнечных батарей, от географического положения электростанции и климатических условий обуславливает значительные различия в характеристиках солнечной радиации для разных регионов России. Соответственно, существенно могут различаться и параметры следящих систем, обеспечивающих максимальную производительность солнечной электростанции. Опыт практического применения солнечных трекеров в России небольшой, и актуальной является задача выбора параметров следящей системы, обеспечивающих максимальную технико-экономическую эффективность фотоэлектрической станции в заданном районе ее эксплуатации.

Цель исследования: выбор параметров и оценка эффективности применения солнечных систем слежения в высоких северных широтах

Методы исследования: математическое и компьютерное моделирование с использованием программной среды МаtLab/Simulink.

Результаты. Рассмотрена классификация и основные компоненты солнечных следящих систем, выполнен анализ их основных технических характеристик. Даны рекомендации по выбору параметров следящих систем для эксплуатации в высоких северных широтах. Разработана имитационная модель фотоэлектрической станции с системой слежения за положением Солнца, обеспечивающая моделирование ее энергетических характеристик с учетом реальных условий эксплуатации. Рассмотрен практический пример выбора параметров и оценки эффективности применения солнечных систем слежения для фотоэлектрической станции, территориально расположенной в г. Томске.

\section{Ключевые слова:}

Солнечная система слежения, солнечный трекер, солнечная радиация, имитационная модель, MatLab, Simulink.

\section{Введение}

Из всех известных способов производства электрической энергии наиболее востребованными и динамично развивающимися в настоящее время являются фотоэлектрические технологии, к конкурентным преимуществам которых относят большой срок службы основных энергетических компонентов, минимальные эксплуатационные затраты, возможность создания генерирующих установок на широкий диапазон мощностей с максимальным приближением к объектам электропотребления.

Однако, несмотря на очевидные достоинства, сдерживающими факторами внедрения фотоэлектрических станций (ФЭС) остается высокая стоимость оборудования и низкая әффективность преобразования энергии в сравнении с другими типами энергетических установок. Это заставляет разработчиков фотоэлектрических систем искать новые и совершенствовать известные технические решения, обеспечивающие повышение эффективности, производительности и снижения себестоимости генерируемой энергии. Одним из таких решений является применение систем слежения за положением Солнца - солнечных трекеров.

Опыт эксплуатации солнечных систем слежения (CCC) в разных странах мира показал, что их применение обеспечивает повышение производительности ФЭС в среднем от 15 до $72 \%$ в зависимости от типа трекера, географического положения электростанции, климатических условий и времени года $[1,2]$. Положительный опыт применения ССС стимулирует развитие данной технологии солнечной энергетики. По данным исследований GTM Research, представленных в докладе «Global PV Tracker Landscape 2016», в текущем году в мире установлено 12,6 ГВт ФЭС, оборудованных солнечными трекерами, а к 2021 г. количество установок для трекеров вырастет до 37,7 ГВт, что составит почти половину всех наземных солнечных станций. По данным исследовательской компании Zion Research, в 2021 г. ожидается, что мировой рынок солнечных фотоэлектрических трекеров достигнет 3682,2 млн долл. США, ежегодно увеличиваясь на 18,6 \% в период между 2016 и 2021 гг.

Необходимо отметить, что существует целый ряд технических и экономических ограничений в использовании ССC, которые должны быть тщательно проанализированы при проектировании ФЭС с целью определения оптимальной конфигурации солнечного трекера для каждого конкретного применения. К экономическим ограничениям следует отнести дополнительные капитальные затра- 
ты на приобретение и монтаж ССС, более высокие затраты на техническое обслуживание электростанции из-за наличия движущихся механических частей, необходимость в дополнительной площади участка для размещения солнечных батарей в сравнении со станциями с жестко закрепленными солнечными панелями. Технические ограничения определяются сравнением заявленных производителем характеристик солнечного трекера с прогнозируемыми условиями его эксплуатации: рабочий диапазон углов слежения по азимуту и наклону, максимальная ветровая нагрузка, используемый метод и алгоритм слежения за положением Солнца, тип климатического исполнения и др.

\section{Классификация и основные компоненты}

\section{солнечных следящих систем}

Современные солнечные трекерные системы весьма разнообразны и могут существенно различаться по стоимости, конструкции и используемым принципам управления. Основные требования к ССС определяет ГОСТ Р 57229-2016 [3] (подготовленный на основе международного стандарта IEC 62817-2014 [4]), в котором установлены основные характеристики систем слежения за положением Солнца, их классификационные признаки и методы испытаний.

По количеству и направлению осей вращения солнечные трекеры классифицируются на одноосевые (single axis trackers - SAT) и двухосевые (dual axis tracker - DAT) [3, 4].

В сравнении с двухосевыми системами одноосевые солнечные трекеры имеют более простую конструкцию, а соответственно и низкую стоимость, благодаря чему они получили наибольшее распространение. В зависимости от конструктивного исполнения одноосевые трекеры подразделяются на трекеры с горизонтальной (HSAT), вертикальной (VSAT), наклонной (TSAT) и с полярно-ориентированной (PSAT) осями вращения [5]. Каждая из конструкций имеет свои достоинства и недостатки, и преимущественную область применения. Для высоких широт, характеризуемых значительными изменениями продолжительности светового дня и небольшими углами высоты Солнца, целесообразно использовать трекеры с вертикальной или наклонной осями вращения, для районов вблизи экватора более эффективны трекеры с горизонтальной осью вращения [6].

Максимальную эффективность использования солнечной энергии обеспечивают двухосевые трекеры, конструкции которых подразделяются по направлению основной оси. Из двухосевых систем слежения наибольшее распространение получили трекеры с осью вращения на несущем столбе (tiptilt dual axis tracker - TTDAT) и трекеры с опорной плоскостью (azimuth-altitude dual axis tracker AADAT), в которых главная ось является вертикальной. Достоинствами TTDAT трекеров является простота конструкции и большая гибкость территориального размещения, что позволяет их ис- пользовать для построения ФЭС как небольшой мощности для индивидуального применения, так и в крупных энергетических проектах. B AADAT трекерах в качестве опорной конструкции используется крупное кольцо, которое устанавливается на ролики или большую платформу с подшипниками. Преимуществом такого конструктивного решения является равномерное распределение веса солнечной батареи по частям кольца, в отличие от одной точки загрузки, как в TTDAT системах. Это позволяет использовать трекеры данного типа в ФЭС с солнечными батареями большой мощности и в районах с высокими ветровыми нагрузками, однако такие системы являются более дорогими и требуют большей территории.

Основными компонентами ССС являются: установочная конструкция или опорная рама, привод, трансмиссия и блок управления [7].

Установочная конструкция предназначена для крепления фотоэлектрических модулей к системе слежения за Солнцем и должна обеспечивать необходимую прочность и жесткость к внешним воздействиям. Основными характеристиками установочной конструкции, которые обязан определить изготовитель в технической спецификации на трекерную систему, является максимально допустимый вес и площадь солнечной батареи, максимальная допустимая скорость ветра в режиме слежения и штормовом положении, диапазон рабочих температур, максимальная снеговая нагрузка.

Привод солнечного трекера обеспечивает перемещение CCC вокруг оси вращения. По типу привода различают системы с электроприводом, гидроприводом или пассивным приводом. В системах слежения с пассивным приводом перемещение подвижной части установочной конструкции вокруг оси обеспечивается за счет перепада давления рабочей жидкости, в гидроприводах для перемещения используются исполнительные гидромоторы или гидроцилиндры, которые преобразуют давление, создаваемое насосами, во вращательное или линейное перемещение.

Наибольшее распространение в солнечных трекерных системах получили электроприводы, в которых в качестве исполнительных устройств применяются электрические машины различного типа: линейные, шаговые, серводвигатели и др. Основными характеристиками электропривода ССС являются: тип исполнительного устройства, его номинальная мощность, параметры напряжения питания, величина потребляемой мощности в режиме слежения, среднесуточное или годовое потребление электроэнергии.

К элементам трансмиссии ССС относят компоненты, которые передают механическое движение от приводных двигателей к подвижным элементам следящей системы. Основными характеристиками трансмиссии являются: угловой диапазон перемещения солнечной батареи по азимуту и наклону, точность позиционирования, скорость перемещения или время поворота на один градус. 
Блок управления обеспечивает позиционирование солнечной батареи на Солнце в соответствии с принятым алгоритмом слежения. Для реализации данной функции в составе блока управления используется разнообразное электронное оборудование: микропроцессор, блоки питания, полупроводниковые преобразователи, пускозащитные аппараты исполнительных механизмов, блоки преобразования и передачи данных и т. п. Основными характеристиками системы управления являются: тип управления и алгоритм слежения, тип и протоколы внешнего сопряжения и др.

На практике применяются два основных алгоритма слежения за положением Солнца - астрономический и по датчикам света [8]. Вследствие простоты технической реализации чаще используется алгоритм слежения по световым датчикам, принцип действия которого заключается в позиционировании солнечной батареи на максимальную интенсивность света на небосводе, определяемую по текущим показаниям фотоприемников. Серьезным недостатком трекерных систем с датчиками света является их неработоспособность в пасмурную погоду, при интенсивных осадках и загрязнении фотоприемников.

Более надежны астрономические алгоритмы, которые в простейшем случае могут быть реализованы путем дискретного изменения положения солнечной батареи по суточной программе таймера. Недостатком такого решения для ФЭС, территориально расположенных в районах с высокой широтой, является необходимость постоянной корректировки суточной программы, так как значения азимутальных углов восхода и заката Солнца, а также зенитный угол, подвержены значительным сезонным изменениям. Наибольшую эффективность обеспечивают алгоритмы солнечной позиции (solar position algorithm - SPA), принцип действия которых основан в программном расчете оптимальных углов позиционирования солнечной батареи по заданным параметрам расположения солнечного трекера: широта, долгота, высота над уровнем моря [9].

Необходимо отметить, что в приведенном выше обзоре кратко представлены только основные характеристики компонентов ССС, на которые следует обратить первоочередное внимание при выборе модели для конкретного применения. Однако существуют и другие характеристики ССС, которые необходимо учитывать при выборе, монтаже и эксплуатации, например место установки (земля, крыша), тип фундамента, режим и время приведения в штормовое положение, масса, размеры, характеристики надежности и т. п.

Подробная классификация ССС рассмотрена в работах [10-12], обзор основных компонентов и методов слежения, используемых в современных трекерных системах, выполнен в работах $[7,11,13]$, результаты сравнительного анализа производительности ФЭС с фиксированными солнечными панелями и оборудованными системой слежения пред- ставлены в [14-17]. Вопросам математического моделирования ССС посвящены работы [18-20], подробный анализ наиболее перспективных направлений исследований в данной предметной области выполнен в [20], результаты проектирования и экспериментального исследования солнечных трекеров различных типов представлены в [21-23], оригинальные способы улучшения конструкции и повышения эффективности алгоритмов слежения предложены в работах [23-25], задачи техникоэкономического анализа эффективности применения ССС рассматриваются в работах $[17,26,27]$.

\section{Определение параметров и анализ энергетических характеристик солнечной следящей системы}

Существенные различия в основных технических характеристиках ССС, представленных на рынке, создают серьезные трудности для конечного потребителя при выборе определенной модели солнечного трекера для конкретного применения.

При выборе модели ССС необходимо обратить особое внимание на технические характеристики, определяющие стойкость к внешним климатическим воздействиям. На рынке представлено множество моделей солнечных трекеров, предназначенных для стран с теплым климатом (диапазон рабочей температуры от -10 до $\left.+50{ }^{\circ} \mathrm{C}\right)$, которые малопригодны для эксплуатации на бо́льшей части территории нашей страны. Также отметим, что в районах с суровыми климатическими условиями нецелесообразно использовать трекерные системы с пассивным типом привода вследствие их невысокой надежности [28].

Важными техническими характеристиками ССС являются рабочие диапазоны перемещения по азимуту и наклону, которые должны покрывать максимально возможные значения углов азимута и высоты Солнца в планируемом месте размещения ФЭС.

Максимальные углы азимута и высоты Солнца в Северном полушарии соответствуют дню летнего солнцестояния и определяются географической широтой местности. Углы восхода $\omega_{\mathrm{sr}}$ и заката $\omega_{\mathrm{ss}}$ Солнца по солнечному времени вычисляются по выражениям:

$$
\begin{aligned}
& \omega_{\mathrm{sr}}=-\arccos [-\operatorname{tg} \varphi \cdot \operatorname{tg} \delta] ; \\
& \omega_{\mathrm{ss}}=\arccos [-\operatorname{tg} \varphi \cdot \operatorname{tg} \delta],
\end{aligned}
$$

где $\varphi$ - широта местности в точке установки приемной площадки; $\delta$ - угол склонения Солнца.

Угол склонения определяется по формуле:

$$
\delta=23.45 \cdot \sin \left[\frac{360}{365}(N+284)\right], \text { град, }
$$

где $N$ - номер календарного дня с начала года.

День летнего солнцестояния - это 21 июня, соответственно $N=171$.

Угол высоты Солнца над горизонтом $h$ определяется по выражению:

$$
h=90-\varphi+\delta, \text { град. }
$$


Азимутальный угол положения Солнца $A z$ находится из уравнения [29]:

$$
A z=\arccos \left(\frac{\sinh \cdot \sin \varphi-\sin \delta}{\cosh \cdot \cos \varphi}\right) .
$$

Подставив в выражение (4) значение угла $h=0^{\circ}$, что соответствует восходу (закату) Солнца, легко определить величину максимального азимутального угла.

Например, для г. Томска (географические координаты $56.5^{\circ}$ с.ш., $85.0^{\circ}$ в.д.) по уравнениям (1)-(4) получены следующие значения углов для дня летнего солнцестояния: азимутальный угол восхода (заката) $A z \approx 136^{\circ}$, угол высоты Солнца в полдень $h \approx 57^{\circ}$. Соответственно для ФЭС, территориально расположенной в районе г. Томска, требуется следящая система со следующими рабочими диапазонами перемещения: $270^{\circ}$ по азимуту, и $60^{\circ}$ по наклону.

При определении параметров солнечной следящей системы удобно использовать диаграмму траектории движения Солнца, внешний вид которой показан на рис. 1.

Данная диаграмма особенно полезна, если рядом с местом размещения ФЭС имеются естественные или искусственные препятствия, частично перекрывающие путь солнечным лучам на приемную площадку. Нанесение угловых размеров препятствий на солнечную диаграмму позволяет определить сектора затенения солнечной батареи, учет которых обеспечивает повышение точности при анализе энергетической эффективности ФЭС. Для построения солнечной диаграммы можно воспользоваться бесплатным онлайн сервисом от SRML (Лаборатория мониторинга солнечного излучения) университета штата Орегон [30].

Диапазон изменения углов азимута и высоты Солнца определяет требования к еще одной важ- ной характеристике $\mathrm{CCC}$ - скорости перемещения приемной поверхности. Для нахождения минимально допустимых скоростей перемещения приемной площадки солнечным трекером нужно построить графики изменения углов высоты $h$ и азимута $A z$ Солнца в функции времени для дня летнего солнцестояния.

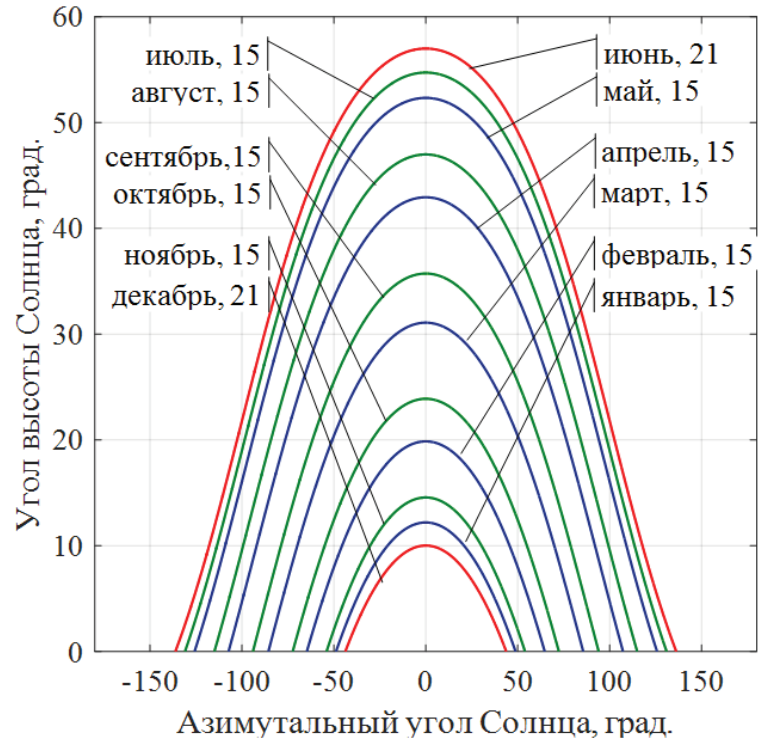

Рис. 1. Диаграмма траектории движения Солнца для города Томска

\section{Fig. 1. Diagram of the Sun motion trajectory in Tomsk}

На рис. 2 представлены графики изменения углов высоты и азимута Солнца в день летнего солнцестояния для двух городов России: Якутска $\left(62^{\circ} 03^{\prime}\right.$ с.ш., $129^{\circ} 27^{\prime}$ в.д.) и Владивостока $\left(43^{\circ} 04^{\prime}\right.$ с.ш., $131^{\circ} 32^{\prime}$ в.д.). Необходимая скорость перемещения определяется крутизной зависимостей углов от времени, и из графиков видно, что для высоких
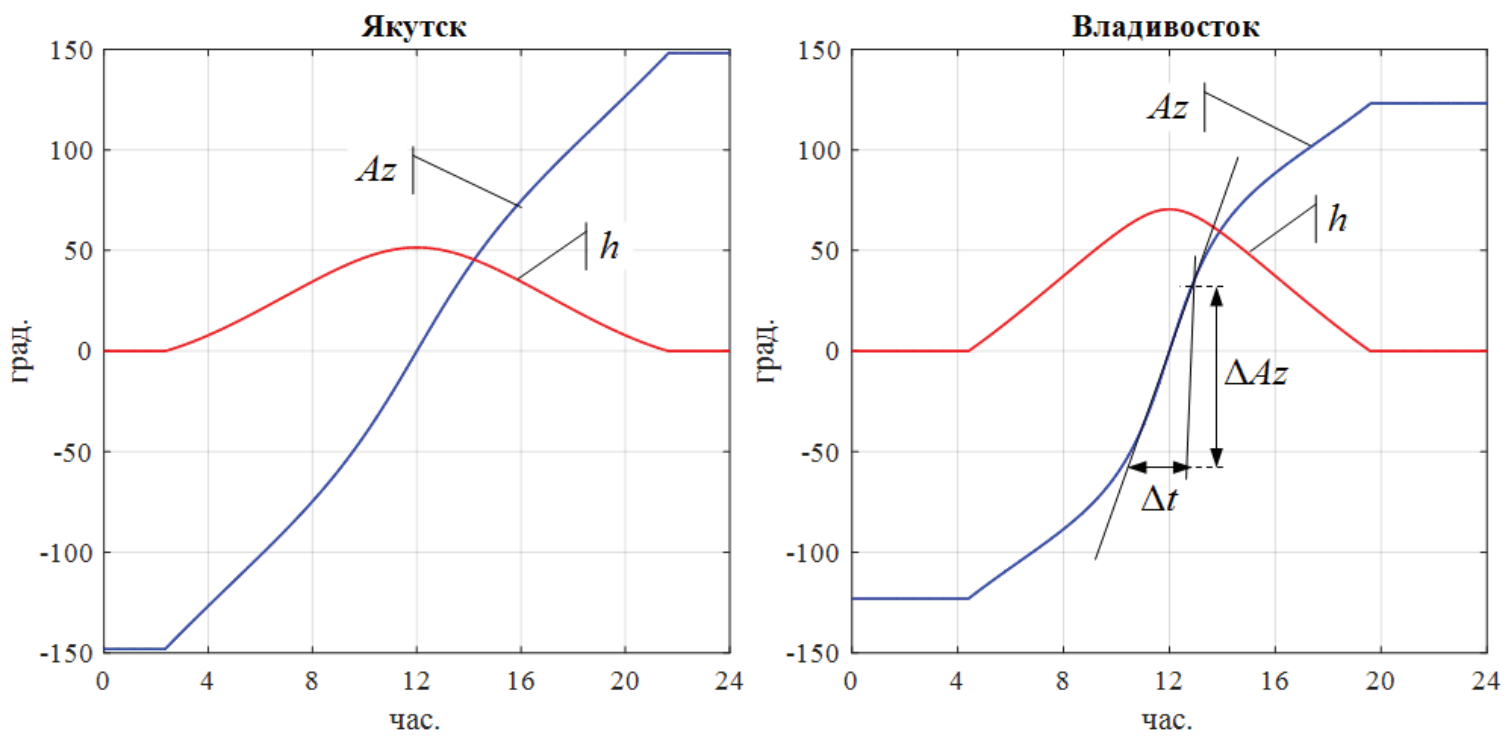

Pис. 2. Графики изменения углов высоты и азимута Солнца в день летнего солнцестояния

Fig. 2. Graphs of changes in the altitude and azimuth of the Sun on the day of the summer solstice 
широт большую скорость перемещения должен обеспечивать привод азимутальной оси.

Для режима непрерывного слежения требуемая угловая скорость азимутального привода $\omega_{\text {пр }}$ определяется из уравнения:

$$
\omega_{\text {і }}=\frac{\Delta A z}{\Delta t}, \text { град / мин. }
$$

Графический способ определения данной скорости для города Владивостока показан на рис. 2. Из рисунка видно, что с уменьшением широты требуется большая скорость перемещения приемной площадки. Например, для приведенного выше примера расчетная скорость перемещения азимутального привода составила $\omega_{\text {пр }}=0,54$ град/мин для Владивостока и $\omega_{\text {пр }}=0,33$ град/мин для Якутска.

На практике способ непрерывного слежения за положением Солнца применяется редко, большее распространение получили дискретные методы регулирования, в которых приемная площадка солнечного трекера перемещается на заданный угол через определенный интервал времени $\Delta t_{i}$ (time interval). Численные значения данного параметра составляют от единиц до нескольких десятков минут, во многих моделях трекеров имеется возможность его непосредственного задания. Величина $\Delta t_{i}$ оказывает непосредственное влияние на энергетическую эффективность ФЭС: при уменьшении $\Delta t$ обеспечивается больший приход энергии на приемную поверхность СБ, но это требует больших затрат энергии на привод. Соответственно, для каждой трекерной системы существует некоторая величина $\Delta t_{i}$, при которой энергетическая эффективность ФЭС будет максимальной. Определить оптимальное значение $\Delta t_{i}$ можно путем решения оптимизационной задачи по критерию максимально возможной выработки электроэнергии солнечной электростанцией за определенный период, обычно год, времени:

$$
W\left(\Delta t_{i}\right)=W_{P V}\left(\Delta t_{i}\right)-W_{E D}\left(\Delta t_{i}\right) \Rightarrow \max , \mathrm{B}_{\mathrm{T}} \cdot \mathrm{ч},
$$

где $W_{P V}\left(\Delta t_{i}\right), W_{E D}\left(\Delta t_{i}\right)$ - интегральные значения энергии, выработанной солнечной батареей и потребленной электроприводом при заданных значениях временного интервала $\Delta t_{i}$, соответственно.

Как правило, потери энергии в приводе следящей системы составляют 2-5\% от генерируемой энергии ФЭС, в связи с чем представляется рациональным выбирать такое максимально возможное значение $\Delta t_{i}$, при котором снижение производительности будет составлять не более $2 \%$.

Для определения величины энергии, генерируемой солнечной батареей, удобно использовать методику, предложенную A.D. Jones, C.P. Underwood [31], согласно которой максимальная выходная мощность СБ определяется по выражению:

$$
P_{P V}=C_{F F} \cdot N_{F M} \cdot \eta_{\text {conv }} \cdot G \cdot \ln \left(10^{6} \cdot G\right) / T_{F M},
$$

где $N_{F M}$ - общее число фотоэлектрических модулей (ФМ) в СБ; $C_{F F}-$ постоянный коэффициент СБ; $\eta_{\text {conv }}$ - КПД преобразователя с контроллером максимальной мощности ФЭС; $G$ - текущий уровень солнечной радиации, Вт/м²; $T_{F M}$ - текущая температура $\Phi$.

В настоящей работе для определения величины коэффициента $C_{F F}$, в отличие от оригинальной методики [31], используется более точное выражение:

$$
\begin{gathered}
C_{F F}=\frac{F F \cdot T_{\text {ref }}}{G_{\text {ref }}} \times \\
\times \frac{\left[I_{S C}+k_{I}\left(T_{F M}-T_{\text {ref }}\right)\right]\left[V_{O C}+k_{V}\left(T_{F M}-T_{\text {ref }}\right)\right]}{\ln \left(10^{6} \cdot G_{\text {ref }}\right)},
\end{gathered}
$$

где $F F$ - коэффициент заполнения вольт-амперной характеристики (BAX) ФМ; $T_{\text {ref }}, G_{\text {ref }}-$ значения температуры и освещенности ФМ при стандартных условиях; $k_{I}, k_{V}$ - температурные коэффициенты тока короткого замыкания и напряжения холостого хода ФМ, соответственно.

Коэффициент заполнения ВАХ ФМ определялся по данным их технической спецификации:

$$
F F=I_{M P P} \cdot V_{M P P} / I_{S C} \cdot V_{O C},
$$

где $I_{M P P}, V_{M P P}$ - паспортные значения тока и напряжения ФМ в точке максимальной мощности при стандартных условиях; $I_{S C}, V_{O C}$ - паспортные значения тока короткого замыкания и напряжения холостого хода ФМ при стандартных условиях.

Характерной особенностью ФЭС, расположенных в высоких северных широтах, является ярковыраженная зависимость их производительности от внешних метеорологических факторов, которые необходимо учитывать при анализе энергетических характеристик электростанции. Наиболее просто определяются среднемесячные и среднесуточные значения температуры окружающего воздуха и скорости ветра, которые являются независимыми величинами от параметров проектируемой ФЭС. Исходными данными для их определения являются статистические данные метеорологических наблюдений, которые легко получить из архивов метеорологических сайтов [32, 33].

Для определения суточного хода температуры воздуха можно использовать выражение:

$$
T(t)=\bar{T}+0,5 \Delta T \cdot \cos \left[2 \pi\left(t_{\text {мест }}-t_{\max }\right) / t_{\text {пер }}\right],
$$

где $\bar{T}$ - среднесуточная температура воздуха, ${ }^{\circ} \mathrm{C}$; $\Delta T$ - суточная амплитуда температуры воздуха, ${ }^{\circ} \mathrm{C}$; $t_{\text {пер }}$ - период изменения температуры воздуха, ч; $t_{\text {max }}$ - местное время максимума температуры, ч; $t_{\text {мест }}$ - локальное (местное) солнечное время, ч.

Кроме окружающего воздуха на температурный режим ФМ оказывают непосредственное влияние: энергия излучения, поступающая на поверхность ФМ вместе с солнечными лучами, и внутренние источники теплоты, образующиеся за счет электрических потерь в солнечных элементах и контактных сопротивлениях. Рабочая температура ФМ при эксплуатации устанавливается в результате теплообмена его поверхности с окружающей средой, имеющего конвективно-лучистый характер. Определение текущей температуры ФМ теоретическими методами практически невозмож- 
но из-за влияния на процесс множества самых разнообразных факторов: коэффициентов теплоотдачи и поглощения излучения материалов модуля, силы и направления ветра, соотношения площадей лицевой и тыльной поверхностей модуля и т. п. Результаты эксплуатации ФЭС показывают, что превышение температуры ФМ над температурой внешней среды при высоких значениях солнечной инсоляции может достигать $30{ }^{\circ} \mathrm{C}$, поэтому учет реальной температуры $Ф \mathrm{M}$ в эксплуатационных условиях является обязательным. В связи со сложностью теоретического определения температуры ФМ используются эмпирические зависимости, полученные в реальных условиях эксплуатации ФЭС [34, 35], адаптированные к определенным климатическим условиям.

Хорошее приближение к реальной зависимости превышения температуры поверхности ФМ над температурой среды дает следующее эмпирическое уравнение:

$$
\Delta T_{F M}=0,0283 G-0,0058 G V+0,0005 G V^{2},{ }^{\circ} \mathrm{C},
$$

где $G$ - величина солнечной радиации, приходящей на поверхность СБ, Вт/м $\mathrm{M}^{2} ; V$ - средняя скорость ветра в месте размещения ФЭС, м/с.

Энергетическая эффективность ССС определяется интенсивностью солнечной радиации, но при этом она во многом зависит не только от величины суммарной радиации, но и от долей ее основных компонент: прямой и рассеянной. Соответственно, ключевой задачей при оценке энергетической эффективности солнечных трекеров является достоверное определение величины солнечной радиации, поступающей на произвольно-ориентированную приемную поверхность.

В настоящей работе используется комбинированная модель солнечной радиации, построенная на базе модели, подробно представленной в [36]. В предложенной модели часть параметров рассчитывается по известным аналитическим выражениям, а часть определяется с помощью эмпирических коэффициентов, полученных из базы данных NASA SSE [37] для заданной в расчетах географической точки размещения гелиоустановки:

$$
\begin{gathered}
G=\left(G_{H}-G_{D H}\right) \frac{\cos \theta}{\cos \theta_{z}}+ \\
+G_{D H}\left[A_{\uparrow} \frac{\cos \theta}{\cos \theta_{z}}+\left(1-A_{1}\right)\left(\frac{1+\cos \beta}{2}\right)\right]+ \\
+G_{H} \rho \frac{1-\cos \beta}{2},
\end{gathered}
$$

где $G_{H}, G_{D H}-$ значения суммарной и рассеянной солнечной радиации, поступающей на горизонтальную поверхность, соответственно; $\theta-$ угол между направлением потока солнечного излучения к поверхности и нормалью к ней; $\theta_{z}-$ зенитный угол Солнца; $\rho$ - альбедо земной поверхности; $\beta$ - угол наклона приемной площадки к горизонтальной плоскости; $A_{i}$ - показатель анизотропии, который определяется по уравнению:

$$
A_{i}=\frac{G_{H}-G_{D H}}{G_{0}},
$$

где $G_{0}$ - внеатмосферная радиация на горизонтальную поверхность.

Для определения значений рассеянной радиации используется анизотропная модель J.Е. Нау [38], которая по результатам исследований, представленных в работе [39], обеспечивает более высокую точность в расчете значений рассеянной солнечной радиации в высоких северных широтах.

На основе представленных выше уравнений была разработана математическая модель солнечной следящей системы, обеспечивающая проведение анализа ее основных энергетических характеристик. Модель реализована в популярном математическом пакете MatLab/Simulink, что обеспечивает простоту и удобство ее практического применения.

\section{Результаты и их обсуждение}

Рассмотрим практическую задачу выбора параметров и оценки эффективности применения солнечной следящей системы для ФЭС, территориально расположенной в г. Томске. Используем для проведения анализа две модели солнечных трекеров от компании Macsun Solar Energy Technology [40], основные технические характеристики которых приведены в таблице.

Из таблицы видно, что технические характеристики выбранных моделей солнечных трекеров в целом удовлетворяют требованиям эксплуатации в заданном районе размещения ФЭС. К сожалению, в технической спецификации производитель не отразил ряд важных характеристик ССС, в связи с чем в расчетах использовались типичные значения этих параметров от других производителей аналогичных моделей $[41,42]$ :

- интервал позиционирования (time interval) 1-30 мин;

- угловая скорость привода - 18 \%мин.

В качестве объекта анализа принята схема ФЭС, в состав которой входит СБ, состоящая из 20 фотоэлектрических модулей поликристаллического типа JАР6-60-260 номинальной мощностью 260 Вт, основные технические характеристики которых представлены на сайте производителя [43].

В качестве исходных данных моделирования использовались метеорологические сводки для г. Томска, полученные по результатам многолетних наблюдений. При выполнении расчетов приняты средние значения температуры окружающего воздуха и скорости ветра, полученные в результате обработки статистических данных за 2005-2016 гг. по метеостанции № 29430 г. Томска, представленные в свободном доступе на сайте «Расписание погоды» [32]. Среднемесячные значения индекса прозрачности атмосферы и альбедо земной поверхности определены из базы данных NASA SSE [37]. 
таблица. Основные технические характеристики солнечных трекеров компании Macsun Solar Energy Technology [40]

Table. The main technical characteristics of solar trackers manufactured by Macsun Solar Energy Technology Co. [40]

\begin{tabular}{|c|c|c|}
\hline \multirow{2}{*}{$\begin{array}{l}\text { Технические характеристики } \\
\text { Product Characteristics }\end{array}$} & \multicolumn{2}{|c|}{ Модель/Model } \\
\hline & MS-PV-SAT33 & MS-PV-SDT33 \\
\hline $\begin{array}{l}\text { Tип системы слежения } \\
\text { Racking/Tracking Type }\end{array}$ & $\begin{array}{l}\text { одноосевая } \\
\text { single axis }\end{array}$ & $\begin{array}{l}\text { двухосевая } \\
\text { dual axis }\end{array}$ \\
\hline $\begin{array}{l}\text { Площадь солнечной батареи } \\
\text { Modules Assembly Area }\end{array}$ & \multicolumn{2}{|c|}{$33 \mathrm{~m}^{2}$} \\
\hline $\begin{array}{l}\text { Количество фотоэлектрических } \\
\text { модулей } \\
\text { Modules Assembly Arrangement }\end{array}$ & \multicolumn{2}{|c|}{20} \\
\hline $\begin{array}{l}\text { Рекомендуемый тип модулей } \\
\text { Modules Reference }\end{array}$ & \multicolumn{2}{|c|}{$260 \mathrm{~W}, 1650 \times 991 \times 40 \mathrm{~mm}$} \\
\hline $\begin{array}{l}\text { Диапазон углов перемещения } \\
\text { по азимуту } \\
\text { Tracking Azimuth Angle }\end{array}$ & \multicolumn{2}{|c|}{ $\pm 120^{\circ}$} \\
\hline $\begin{array}{l}\text { Диапазон углов перемещения } \\
\text { по наклону } \\
\text { Tracking Altitude Angle }\end{array}$ & - & $10-70^{\circ}$ \\
\hline $\begin{array}{l}\text { Диапазон рабочей температуры } \\
\text { Working Temperature }\end{array}$ & \multicolumn{2}{|c|}{$-40+60^{\circ} \mathrm{C}$} \\
\hline $\begin{array}{l}\text { Максимальная допустимая } \\
\text { скорость ветра в режиме } \\
\text { слежения } \\
\text { Max. Operating Wind Load }\end{array}$ & \multicolumn{2}{|c|}{$22 \mathrm{~m} / \mathrm{s}$} \\
\hline $\begin{array}{l}\text { Максимальная допустимая } \\
\text { скорость ветра в штормовом } \\
\text { положении } \\
\text { Max. Wind Load at Stow Position }\end{array}$ & \multicolumn{2}{|c|}{$34 \mathrm{~m} / \mathrm{s}$} \\
\hline $\begin{array}{l}\text { Точность слежения } \\
\text { Tracking Accuracy }\end{array}$ & \multicolumn{2}{|c|}{$\leq 1^{\circ}$} \\
\hline $\begin{array}{l}\text { Тип привода } \\
\text { Tyре of Drive }\end{array}$ & \multicolumn{2}{|c|}{$\begin{array}{l}\text { электрический } \\
\text { electrical }\end{array}$} \\
\hline $\begin{array}{l}\text { Мощность привода } \\
\text { Motor Power }\end{array}$ & \multicolumn{2}{|c|}{$96 \mathrm{~W}$} \\
\hline \begin{tabular}{|l|} 
Алгоритм слежения \\
за положением Солнца \\
Tracking Principle Algorithm
\end{tabular} & \multicolumn{2}{|c|}{ SPA } \\
\hline
\end{tabular}

Для сокращения объема вычислений при выполнении расчетов использовался метод «средних суток месяца», предложенный S.A. Klein [44], согласно которому среднесуточная интенсивность солнечной радиации за рассматриваемый месяц года равна суточной интенсивности радиации для соответствующего рекомендованного дня. В соответствии с данным методом, интегральные значения среднемесячной выработки электроэнергии ФЭС определялись простым произведением суточного объема на число дней в соответствующем месяце.

На рис. 3 представлены расчетные значения среднесуточной выработки электроэнергии ФЭС при трех различных вариантах систем слежения (суммарный коэффициент полезного действия преобразователей для всех вариантов принят неизменным и равным 0,85).

Результаты расчетов показывают, что солнечные трекеры обеспечивают повышение производительности ФЭС, однако их эффективность существенно зависит от сезонов года. При использовании одноосевой системы слежения выработка электроэнергии ФЭС увеличивается от 40 до $60 \%$ в летние месяцы года, и от 5 до $12 \%$ в зимние. Для двухосевой системы слежения данные показатели составляют от 44 до $67 \%$ для летних месяцев, и от 12 до $16 \%$ для зимних. В годовом выражении применение одноосевой системы слежения позволяет повысить потенциальную производительность ФЭС на $35 \%$, а двухосевой системы на $39 \%$.

Незначительная разница в энергетической эффективности одноосевой и двухосевой систем слежения объясняется тем, что в высоких северных широтах величина угла высоты Солнца в течение светового дня изменяется в относительно небольших пределах, особенно в зимние месяцы года. Соответственно, для оптимального позиционирования поверхности СБ на Солнце (величина угла $\theta=0^{\circ}$ ) требуется изменять ее наклон в достаточно узком диапазоне углов.

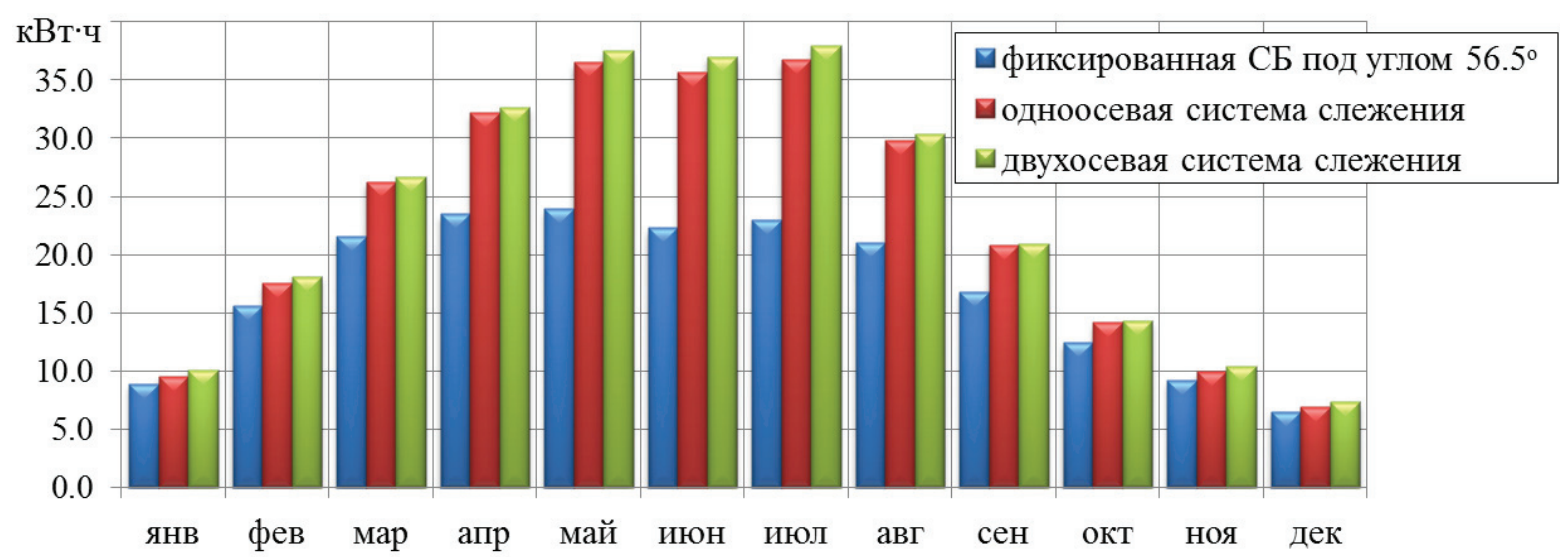

Рис. 3. Расчетные значения среднесуточной выработки электроэнергии ФЭС с различными системами слежения за положением Солнца

Fig. 3. Calculated values of the daily average power output from photovoltaic plants with different solar tracking systems 

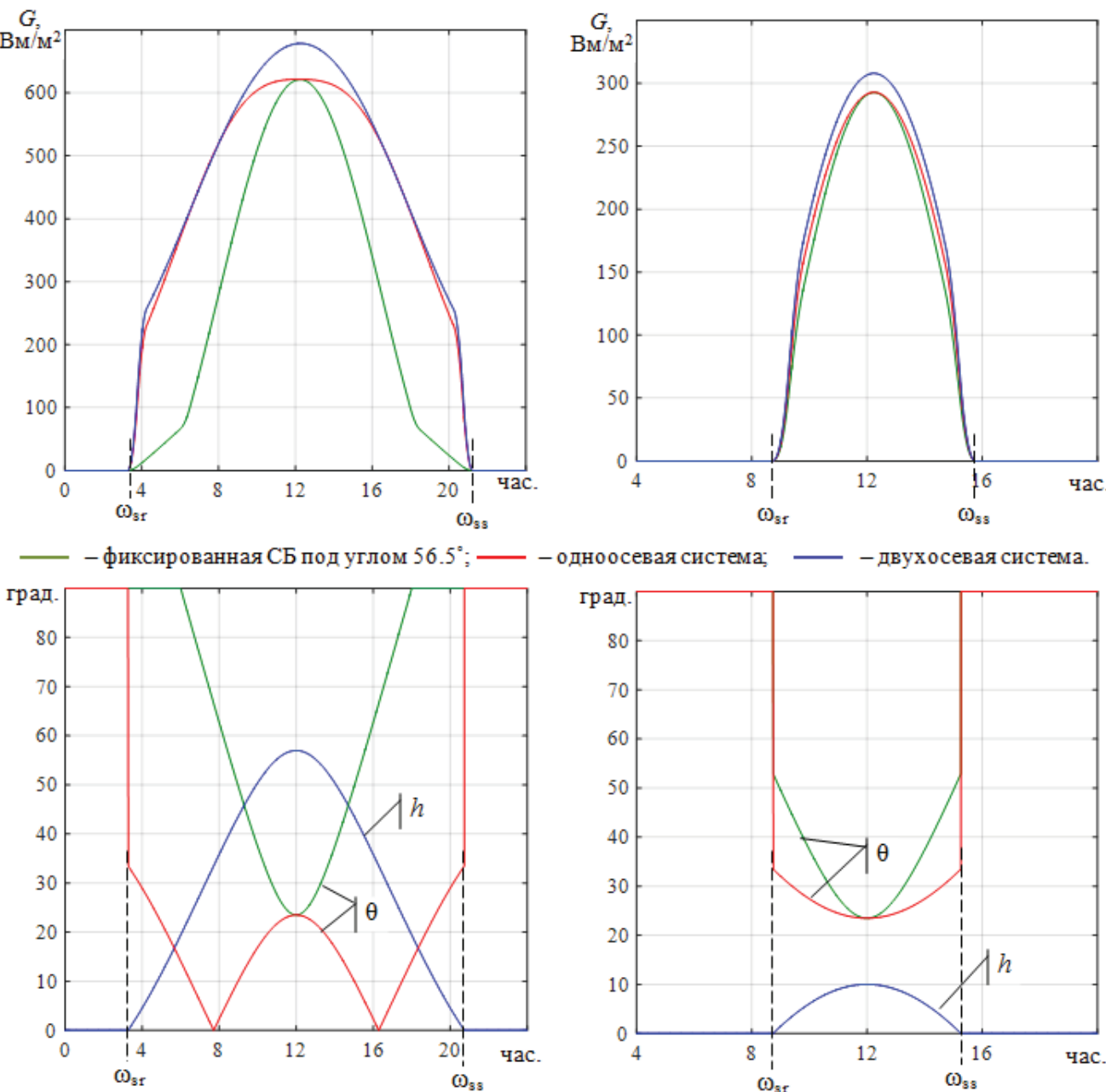

одноосевая система; - - двухосевая система.

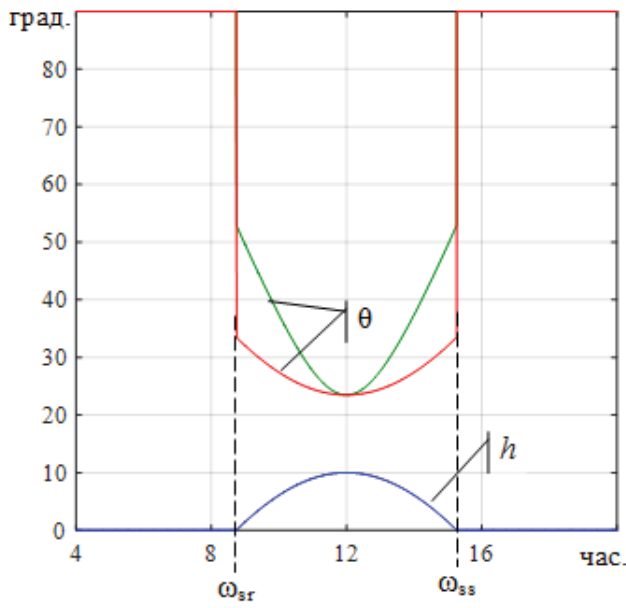

— - фиксированная СБ под углом $56.5^{\circ}$; - - одноосевая система

Рис. 4. Суточные изменения суммарной солнечной радиации G, приходящей на поверхность солнечной батареи, и значения углов һ и $\theta$

Fig. 4. Daily changes in the total solar radiation $G$ arriving to the surface of the solar panel, and the values of angles $h$ and $\theta$

На рис. 4 представлены расчетные зависимости прихода суммарной солнечной радиации $G$ на поверхность СБ при использовании различных ССС для двух характерных дней года: летнего и зимнего солнцестояния. На этом же рисунке показаны изменения угла высоты Солнца $h$ и угла $\theta$ для жестко зафиксированной под углом широты местности и ориентированной на Юг СБ, а также для азимутального солнечного трекера (при применении двухосевой непрерывной системы слежения величина угла $\theta=0^{\circ}$ ).

Из графических зависимостей, представленных на рис. 4 , хорошо видно, что для дня летнего солнцестояния величина отклонения угла $\theta$ от оптимального значения $0^{\circ}$ для фиксированной СБ составляет от 90 до $23,5^{\circ}$, достигая максимума в часы суток, близких к рассвету и закату. Азимутальный солнечный трекер позволяет сузить данный диапазон углов до значений от 33,5 до $0^{\circ}$, чем обеспечивается существенное увеличение значений прямой солнечной радиации, приходящей на по- верхность СБ. В сравнении с двухосевой системой слежения максимальные потери прямой солнечной радиации азимутального трекера составят не более $17 \%\left(\cos 33,5^{\circ}=0,83\right)$, а с учетом того, что эти потери наблюдаются в послерассветные и предзакатные часы, в абсолютном выражении они будут несущественными.

В день зимнего солнцестояния продолжительность светового дня на широте $56.5^{\circ}$ составляет менее 6 часов при максимальном угле высоты Солнца около $10^{\circ}$, что практически нивелирует эффективность применения следящих систем (рис. 4).

Для оценки влияния величины интервала позиционирования $\Delta t_{i}$ на энергетическую эффективность ФЭС была проведена серия вычислительных экспериментов по определению интенсивности суммарной солнечной радиации, поступающей на приемную поверхность СБ, для режима непрерывного слежения и трех значений $\Delta t_{i}=5 ; 15$ и 30 мин. Результаты расчетов показали, что для всех принятых значений интервала позиционирования 
уменьшение интенсивности солнечной радиации составляет не более 1 \% в сравнении с системой непрерывного слежения, что определяет выбор рационального значения $\Delta t_{i}=30$ мин.

На рис. 5 представлены расчетные значения углов позиционирования СБ (азимутальный угол $A z$, угол наклона $E l)$ двухосевой системой слежения в день летнего солнцестояния с интервалом $\Delta t_{i}=30$ мин. На этом же рисунке показаны суточные изменения величины угла $\theta$.

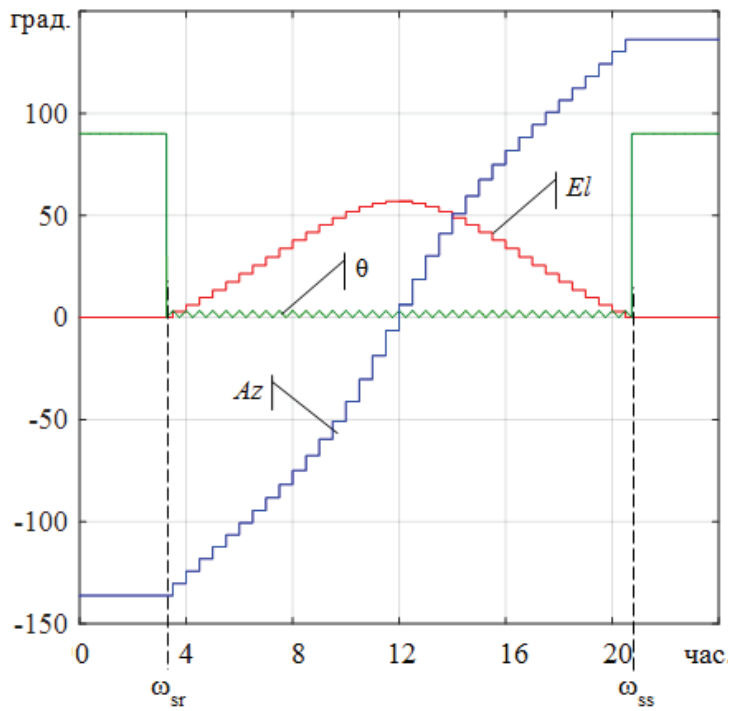

Рис. 5. Углы позиционирования СБ двухосевой системой слежения в день летнего солнцестояния

Fig. 5. Angles of positioning the solar panel with a dual-axis tracking system on the day of the summer solstice

Анализ полученных зависимостей показывает, что при принятых параметрах ССС предельные отклонения угла $\theta$ от оптимального значения соста- вляют не более $3,5^{\circ}$, что обеспечивает практически максимальную производительность ФЭС.

На основании проведенных исследований можно сделать следующие выводы:

- применение ССС в высоких северных широтах позволяет увеличить производительность ФЭС на $35-40 \%$;

- в большинстве практических случаев в высоких северных широтах целесообразно использовать более надежные одноосевые системы слежения, так как применение более дорогих двухосевых систем дает незначительный выигрыш в объемах генерируемой электроэнергии;

- в солнечных трекерах, использующих для позиционирования СБ алгоритмы солнечной позиции, целесообразно устанавливать максимально возможные значения интервала позиционирования.

\section{Заключение}

Предложена оригинальная методика выбора параметров и оценки эффективности применения систем слежения за положением Солнца, построенная на базе имитационной модели ФЭС, обеспечивающей расчет ее основных энергетических характеристик с учетом реальных условий эксплуатации.

Результаты исследований могут быть полезны широкому кругу специалистов, занимающихся вопросами проектирования и эксплуатации фотоэлектрических станций.

Работа выполнена при финансовой поддержке Министерства образования и науки Российской Федерации. Уникальный идентификатор работ - RFMEFI57617Х0098 (соглашение № 14.576.21.0098 о предоставлении субсидии от 26 сентября 2017 года).

\section{СПИСОК ЛИТЕРАТУРЫ}

1. Lee J.F., Rahim N.A. Performance Comparison of Dual-Axis Solar Tracker vs Static Solar System in Malaysia // IEEE Conference on Clean Energy and Technology (CEAT). - 2013. P. 102-107.

2. Ribeiro D.B.S., Demetino G.G., Pepe I.M. Solar Trackers: Worldwide Map of Performances // $22^{\text {nd }}$ International Congress of Mechanical Engineering. - Ribeirão Preto, Brazil, 2013. P. 5521-5530.

3. ГОСТ Р 57229-2016. Системы фотоэлектрические. Устройства слежения за Солнцем. Технические условия. - М.: Стандартинформ, 2016. - 64 с.

4. IEC 62817. Photovoltaic systems. Design qualification of solar trackers. ICS 27.160, ISBN 978-2-8322-1826-6.

5. Racharla S., Rajan K. Solar tracking system - a review // International Journal of Sustainable Engineering, - 2017. - V. 10. P. 72-81.

6. Ya'u M.J. A Review on Solar Tracking Systems and Their Classifications // Journal of Energy, Environmental \& Chemical Engineering. - 2017. - V. 2 (3). - P. 46-50.

7. Prinsloo G., Dobson R. Solar Tracking. South Africa. E-Book. 2015. - ISBN: 978-0-620-61576-1. URL: https://www.researchgate.net/profile/Gerro_Prinsloo/ (дата обращения: 08.06.2018)
8. Tracking strategy for photovoltaic solar systems in high latitudes / G. Quesada, L. Guillon, D.R. Rousse et al. // Energy Conversion and Management. - 2015. - V. 103. - P. 147-156.

9. Design of two axes sun tracking controller with analytically solar radiation calculations / S. Yilmaz, H.R. Ozcalik, 0. Dogmus et al. // Renewable and Sustainable Energy Reviews. - 2015. - V. 43. P. $997-1005$.

10. A review of principle and sun-tracking methods for maximizing solar systems output / H. Mousazadeh, A. Keyhani, A. Javadi et al. // Renewable and Sustainable Energy Reviews. - 2009. V. 13. - P. 1800-1818.

11. Recent advancements and challenges in Solar Tracking Systems (STS): a review / W. Nsengiyumva, S.G. Chen, L. Hu, X. Chen // Renewable and Sustainable Energy Reviews. - 2018. - V. 81. P. 250-279.

12. Al-Rousan N., Mat Isa N.A., Mat Desa M.K. Advances in solar photovoltaic tracking systems: a review // Renewable and Sustainable Energy Reviews. - 2018. - V. 82 (3). - P. 2548-2569.

13. Anyaka B.O., Ahiabuike D.C., Mbunwe M.J. Improvement of Pv Systems Power Output Using Sun-Tracking Techniques // International Journal of Computational Engineering Research. 2013. - V. 3 (9). - P. 80-98.

14. Comparative performance analysis between static solar panels and single-axis tracking system on a hot climate region near to 
the equator / R.G. Vieira, F.K.O.M.V. Guerra, M.R.B.G. Vale, M.M. Araújo // Renewable and Sustainable Energy Reviews. 2016. - V. 64. - P. 672-681.

15. Comparison of performance of solar photovoltaics on dual axis tracker with fixed axis at 13?N latitude / R.R. Rao, H.R. Swetha, J. Srinivasan, S.K. Ramasesha // Current science. - 2015. V. 108 (11). - P. 2087-2094.

16. Comparative analysis of fixed and sun tracking low power PV systems considering energy consumption / G.C. Lazaroiu, M. Longo, M. Roscia, M. Pagano // Energy Conversion and Management. - 2015. - V. 92. - P. 143-148.

17. Axaopoulos P.J., Fylladitakis E.D. Energy and economic comparative study of a tracking vs. a fixed photovoltaic system // European Scientific Journal. - 2013. - V. 9 (12). - P. 50-69.

18. Mathematical study of the movement of solar tracking systems based on rational models / L.M. Fernández-Ahumada, F.J. Casares, J. Ramírez-Faz, R. López-Luque // Solar Energy. - 2017. V. $150 .-$ P. $20-29$

19. Bahrami A., Okoye C.0., Atikol U. The effect of latitude on the performance of different solar trackers in Europe and Africa // Applied Energy. - 2016. - V. 177. - P. 896-906.

20. Tilt and azimuth angles in solar energy applications - a review / A.Z. Hafeza, A. Solimana, K.A. El-Metwallya, I.M. Ismaila // Renewable and Sustainable Energy Reviews. - 2017. - V. 77. P. $147-168$.

21. Algarín C.R., Castro A.0., Naranjo J.C. Dual-Axis Solar Tracker for Using in Photovoltaic Systems // International Journal of Renewable Energy Research. - 2017. - V. 7. - P. 137-145.

22. Dual-axis photovoltaic tracking system e Design and experimental investigation / S. Seme, G. Srpcic, D. Kavsek et al. // Energy. - 2017. - V. 139. - P. 1267-1274.

23. Monthly profile analysis based on a two-axis solar tracker proposal for photovoltaic panels / F.M. Hoffmann, R.F. Molz, J.V. Kothe et al. // Renewable Energy. - 2018. - V. 115. P. $750-759$.

24. Automated positioning dual-axis solar tracking system with precision elevation and azimuth angle control / M.H.M. Sidek, N. Azis, W.Z.W. Hasan, M.Z.A. Ab Kadir, S. Shafie, M.A.M. Radzi // Energy. - 2017. - V. 124. - P. 160-170.

25. RAST: Round about Solar Tracking / M. Rosa-Clot, P. Rosa-Clot, G.M. Tina, C. Ventura // Energy Procedia. - 2017. - V. 134. P. 598-606.

26. Simón-Martín M., Alonso-Tristán C., Díez-Mediavilla M. Performance Indicators for Sun-Tracking Systems: A Case Study in Spain // Energy and Power Engineering. - 2014. - V. 6. P. 292-302.

27. Bahrami A., Okove C.0., Atikol U. Technical and economic assessment of fixed, single and dual-axis tracking PV panels in low latitude countries // Renewable Energy. - 2017. - V. 113. P. 563-579.
28. A Review on Photovoltaic Systems: Mechanisms and Methods for Irradiation Tracking and Prediction / H. Loschi, Y. Iano, J. León, A. Moretti, F. Conte, H. Braga / Smart Grid and Renewable Energy, - 2015. - V. 6. - P. 187-208.

29. Duffie J.A., Beckman W.A. Solar Engineering of Thermal Processes. $4^{\text {th }}$ ed. - Hoboken, New Jersey: John Wiley \& Sons, Inc., 2013. $-910 \mathrm{p}$

30. Solar Radiation Monitoring Laboratory Department of Physics, University of Oregon. URL: http://solardat.uoregon.edu/ (дата обращения: 08.10.2018).

31. Jones A.D., Underwood C.P. A thermal model for photovoltaic systems // Solar Energy. -2001. - V. 70 (4). - P. 349-359.

32. Российский метеорологический сайт «Расписание погоды». URL: http://www.rp5.ru/ (дата обращения: 30.03.2018).

33. Сайт «Погода России. Архив погоды». URL: http://meteo.infospace.ru/ (дата обращения: 30.03.2018).

34. Al Riza D.F., Haq Gilani S.I., Aris M.S. Hourly Solar Radiation Estimation Using Ambient Temperature and Relative Humidity Data // International Journal of Environmental Science and Development. - 2011. - V. 2 (3). - P. 188-193.

35. Muzathik A.M. Photovoltaic Modules Operating Temperature Estimation Using a Simple Correlation // International Journal of Energy Engineering. - 2014. - V. 4 (4). - P. 151-158.

36. Обухов С.Г., Плотников И.А. Математическая модель прихода солнечной радиации на произвольно-ориентированную поверхность для любого региона России // Международный научный журнал Альтернативная энергетика и Экология. 2017. - № 16-18 (228-230). - C. 43-56.

37. The NASA Surface Meteorology and Solar Energy Data Set. URL: http://eosweb.larc.nasa.gov/sse/ (дата обращения: 30.03.2018).

38. Hay J.E. Calculation of monthly mean solar radiation for horizontal and inclined surfaces // Solar Energy. - 1979. - V. 23 (4). P. 301-307.

39. Стадник В.В., Разгоняев Ю.В. Оценка суммарного прихода солнечной радиации, поступающей на наклонные поверхности // Труды главной геофизической обсерватории им. А.И. Воейкова. - 2008. - Вып. 557. - С. 67-84.

40. Официальный сайт Macsun Solar Energy Technology Co. URL: http://www.macsunsolar.com/ (дата обращения: 30.03.2018).

41. Официальный сайт Sat Control. URL: http://www.sat-control.net/ (дата обращения: 30.03.2018)

42. Официальный сайт Compañía Española de Alta Eficiencia Fotovoltaica BSQ Solar. URL: https://www.holaluz.com/ (дата обращения: 30.03.2018).

43. Сайт компании «JA SOLAR». URL: http://www.jasolar.com/ (дата обращения: 30.03.2019).

44. Klein S.A. Calculation of monthly average insolation on tilted surfaces // Solar Energy. - 1977. - V. 19 (4). - P. 325-329.

Поступила 15.06.2018 2.

\section{Информация об авторах}

Обухов С.Г., доктор технических наук, профессор отделения электроэнергетики и электротехники Инженерной школы энергетики Национального исследовательского Томского политехнического университета.

Плотников И.А., кандидат технических наук, доцент отделения электроэнергетики и электротехники Инженерной школы энергетики Национального исследовательского Томского политехнического университета. 
UDC 621.311.25: 621.311.29

\title{
CHOOSING THE PARAMETERS AND ANALYZING THE EFFICIENCY OF SOLAR TRACKING SYSTEMS
}

\author{
Sergey G. Obukhov', \\ serob99@mail.ru \\ Igor A. Plotnikov', \\ igorplt@tpu.ru \\ 1 National Research Tomsk Polytechnic University, \\ 30, Lenin Avenue, Tomsk, 634050, Russia.
}

Relevance. Solar tracking systems are the effective way to increase the efficiency of photovoltaic plants. The obvious dependence of the amount of solar radiation arriving at the surface of solar panels on geographic location of the photovoltaic plant and climatic conditions shows significant differences in the characteristics of solar radiation for different regions of Russia. Thus, the parameters of tracking systems that provide the maximum efficiency of solar power plants can vary significantly. So far, solar tracking systems have not been widespread in Russia. Therefore, choosing the parameters of tracking systems is crucial to ensure the maximum technical and economic efficiency of photovoltaic stations in a given area.

The aim of the study is to choose the parameters and analyze the efficiency of solar tracking systems in high northern latitudes.

Research methods: mathematical and computer modeling using the Matlab/Simulink software environment.

Results. The paper considers classification and main components of solar tracking systems; their main technical characteristics are analyzed. The authors give the recommendations for choosing the parameters of a tracking system to provide its proper operation in high northern latitudes. A simulation model of the photovoltaic station with a solar tracking system was developed to enable modeling of its energy characteristics with respect to actual operating conditions. The paper provides a practical example of choosing the parameters and evaluating the efficiency of solar tracking systems for photovoltaic station located in Tomsk. The results of the study can be of value to a wide range of specialists dealing with the design and operation of photovoltaic plants.

\section{Key words:}

Solar tracking system, solar tracker, solar radiation, simulation model, MatLab, Simulink.

The work was supported by the Ministry of Education and Science of the Russian Federation. The unique identifier is RFMEFI57617X0098 (agreement No. 14.576.21.0098 dated September 26, 2017).

\section{REFERENCES}

1. Lee J.F., Rahim N.A. Performance Comparison of Dual-Axis Solar Tracker vs Static Solar System in Malaysia. IEEE Conference on Clean Energy and Technology (CEAT), 2013, pp. 102-107.

2. Ribeiro D.B.S., Demetino G.G., Pepe I.M. Solar Trackers: Worldwide Map of Performances. $22^{n d}$ International Congress of Mechanical Engineering. Ribeirão Preto, Brazil, 2013. pp. $5521-5530$.

3. GOST R 57229-2016. Sistemy fotoelektricheskie. Ustroystva slezheniya za Solntsem. Tekhnicheskie usloviya [State Standard Russia 57229-2016. Photovoltaic systems. Solar trackers. General specifications]. Moscow, Standartinform Publ., 2016. 64 p.

4. IEC 62817. Photovoltaic systems. Design qualification of solar trackers. ICS 27.160, ISBN 978-2-8322-1826-6.

5. Racharla S., Rajan K. Solar tracking system - a review. Interna tional Journal of Sustainable Engineering, 2017, vol. 10, pp. 72-81.

6. Ya'u M.J. A Review on Solar Tracking Systems and Their Classifications. Journal of Energy, Environmental \& Chemical Engine ering, 2017, vol. 2 (3), pp. 46-50.

7. Prinsloo G., Dobson R. Solar Tracking. South Africa. E-Book. 2015. - ISBN: 978-0-620-61576-1. Available at: https://www. researchgate.net/profile/Gerro_Prinsloo/ (accessed 08 June 2018).

8. Quesada G., Guillon L., Rousse D.R. Tracking strategy for photovoltaic solar systems in high latitudes. Energy Conversion and Management, 2015, vol. 103, pp. 147-156.

9. Yilmaz S., Ozcalik H.R., Dogmus 0. Design of two axes sun tracking controller with analytically solar radiation calculations. Renewable and Sustainable Energy Reviews, 2015, vol. 43, pp. 997-1005.
10. Mousazadeh H., Keyhani A., Javadi A. A review of principle and sun-tracking methods for maximizing solar systems output. Renewable and Sustainable Energy Reviews, 2009, vol. 13, pp. $1800-1818$.

11. Nsengiyumva W., Chen S.G., Hu L., Chen X. Recent advancements and challenges in Solar Tracking Systems (STS): a review. Renewable and Sustainable Energy Reviews, 2018, vol. 81, pp. 250-279.

12. Al-Rousan N., Mat Isa N.A., Mat Desa M.K. Advances in solar photovoltaic tracking systems: a review. Renewable and Sustainable Energy Reviews, 2018, vol. 82 (3), pp. 2548-2569.

13. Anyaka B.O., Ahiabuike D.C., Mbunwe M.J. Improvement of Pv Systems Power Output Using Sun-Tracking Techniques. International Journal of Computational Engineering Research, 2013, vol. 3 (9), pp. 80-98.

14. Vieira R.G., Guerra F.K.O.M.V, Vale M.R.B.G., Araújo M.M. Comparative performance analysis between static solar panels and single-axis tracking system on a hot climate region near to the equator. Renewable and Sustainable Energy Reviews, 2016, vol. 64 , pp. 672-681.

15. Rao R.R., Swetha H.R., Srinivasan J., Ramasesha S.K. Comparison of performance of solar photovoltaics on dual axis tracker with fixed axis at 13?N latitude. Current science, 2015, vol. 108 (11), pp. 2087-2094.

16. Lazaroiu G.C., Longo M., Roscia M., Pagano M. Comparative analysis of fixed and sun tracking low power PV systems considering energy consumption. Energy Conversion and Management, 2015, vol. 92 , pp. 143-148.

17. Axaopoulos P.J., Fylladitakis E.D. Energy and economic comparative study of a tracking vs. a fixed photovoltaic system. European Scientific Journal, 2013, vol. 9 (12), pp. 50-69. 
18. Fernández-Ahumada L.M., Casares F.J., Ramírez-Faz J., LópezLuque R. Mathematical study of the movement of solar tracking systems based on rational models. Solar Energy, 2017, vol. 150, pp. $20-29$.

19. Bahrami A., Okoye C.0., Atikol U. The effect of latitude on the performance of different solar trackers in Europe and Africa. Applied Energy, 2016, vol. 177, pp. 896-906.

20. Hafeza A.Z., Solimana A. El-Metwallya K.A. Ismaila I.M. Tilt and azimuth angles in solar energy applications - a review. Renewable and Sustainable Energy Reviews, 2017, vol. 77, pp. $147-168$.

21. Algarín C.R., Castro A.O., Naranjo J.C. Dual-Axis Solar Tracker for Using in Photovoltaic Systems. International Journal of Renewable Energy Research, 2017, vol. 7, pp. 137-145.

22. Seme S., Srpcic G., Kavsek D. Dual-axis photovoltaic tracking system e Design and experimental investigation. Energy, 2017, vol. 139, pp. 1267-1274.

23. Hoffmann F.M., Molz R.F., Kothe J.V. Monthly profile analysis based on a two-axis solar tracker proposal for photovoltaic panels. Renewable Energy, 2018, vol. 115, pp. 750-759.

24. Sidek M.H.M., Azis N., Hasan W.Z.W, Ab Kadir M.Z.A., Shafie S., Radzi M.A.M. Automated positioning dual-axis solar tracking system with precision elevation and azimuth angle control. Energy, 2017, vol. 124, pp. 160-170.

25. Rosa-Clot M., Rosa-Clot P., Tina G.M., Ventura C. RAST: Round about Solar Tracking. Energy Procedia, 2017, vol. 134, pp. 598-606.

26. Simón-Martín M., Alonso-Tristán C., Díez-Mediavilla M. Performance Indicators for Sun-Tracking Systems: a Case Study in Spain. Energy and Power Engineering, 2014, vol. 6, pp. 292-302.

27. Bahrami A., Okoye C.0., Atikol U. Technical and economic assessment of fixed, single and dual-axis tracking PV panels in low latitude countries. Renewable Energy, 2017, vol. 113, pp. 563-579.

28. Loschi, H., Iano Y., León J., Moretti A., Conte F., Braga H. A Review on Photovoltaic Systems: Mechanisms and Methods for Irradiation Tracking and Prediction. Smart Grid and Renewable Energy, 2015, vol. 6, pp. 187-208.

29. Duffie J.A., Beckman W.A. Solar Engineering of Thermal Processes. $4^{\text {th }}$ ed. Hoboken, New Jersey, John Wiley \& Sons, Inc., $2013.910 \mathrm{p}$.

30. Solar Radiation Monitoring Laboratory Department of Physics, University of Oregon. Available at: http://solardat.uoregon.edu/ (accessed 08 October2018).
31. Jones A.D., Underwood C.P. A thermal model for photovoltaic systems. Solar Energy, 2001, vol. 70 (4), pp. 349-359.

32. Rossiysky meteorologichesky sayt "Raspisanie pogody» [Russian meteorological site "Weather Schedule»]. Available at: http://www.rp5.ru/ (accessed 30 March 2018).

33. Sayt «Pogoda Rossii. Arkhiv pogody» [Site «Weather of Russia. Weather archive»]. Available at: http://meteo.infospace.ru/ (accessed 30 March 2018).

34. Al Riza D.F., Haq Gilani S.I., Aris M.S. Hourly Solar Radiation Estimation Using Ambient Temperature and Relative Humidity Data. International Journal of Environmental Science and Development, 2011, vol. 2 (3), pp. 188-193.

35. Muzathik A.M. Photovoltaic Modules Operating Temperature Estimation Using a Simple Correlation. International Journal of Energy Engineering, 2014, vol. 4 (4), pp. 151-158.

36. Obukhov S.G., Plotnikov I.A. Mathematical model of solar radiation incident on an arbitrarily oriented surface for any region in Russia. Alternative Energy and Ecology (ISJAEE), 2017, no. $16-18$, pp. $43-56$. In Rus.

37. The NASA Surface Meteorology and Solar Energy Data Set. Available at: http://eosweb.larc.nasa.gov/sse/ (accessed $30 \mathrm{March}$ 2018).

38. Hay J.E. Calculation of monthly mean solar radiation for horizontal and inclined surfaces. Solar Energy, 1979, vol. 23 (4), pp. 301-307.

39. Stadnik V.V., Razgonyaev Y.V. Otsenka summarnogo prikhoda solnechnoy radiatsii, postupayushchey na naklonnye poverkhnosti [Estimation of the total solar radiation arrival on inclined surfaces]. Trudy glavnoy geofizicheskoy observatorii im. A.I. Voeykova, 2008, vol. 557, pp. 67-84.

40. Macsun Solar Energy Technology Co. Available at: http://www.macsunsolar.com/ (accessed 30 March 2018).

41. Sat Control. Available at: http://www.sat-control.net/ (accessed 30 March 2018).

42. Compañía Española de Alta Eficiencia Fotovoltaica BSQ Solar. Available at: https://www.holaluz.com/ (accessed 30 March 2018).

43. JA SOLAR Co. Available at: http://www.jasolar.com/ (accessed 30 March 2018).

44. Klein S.A. Calculation of monthly average insolation on tilted surfaces. Solar Energy, 1977, vol. 19 (4), pp. 325-329.

Received: 15 June 2018.

\section{Information about the authors}

Sergey G. Obukhov, Dr. Sc., professor, National Research Tomsk Polytechnic University.

Igor A. Plotnikov, Cand. Sc. associate professor, National Research Tomsk Polytechnic University. 